\title{
Long-Term Effectiveness of Direct CT-Guided Aspiration and Fenestration of Symptomatic Lumbar Facet Synovial Cysts
}

\author{
(D).N. Shah, (D) N.D. von Fischer, DC.T. Chin, (DE.L. Yuh, DM.R. Amans, DW.P. Dillon, and (D).P. Hess
}

\begin{abstract}
BACKGROUND AND PURPOSE: Lumbar facet synovial cysts are commonly seen in facet degenerative arthropathy and may be symptomatic when narrowing the spinal canal or compressing nerve roots. The purpose of this study was to analyze the safety, effectiveness, and long-term outcomes of direct CT-guided lumbar facet synovial cyst aspiration and fenestration for symptom relief and for obviating an operation.
\end{abstract}

MATERIALS AND METHODS: We retrospectively reviewed the medical records and imaging studies of 64 consecutive patients between 2006 and 2016 who underwent 85 CT-guided lumbar facet synovial cyst fenestration procedures in our department. We recorded patient demographics, lumbar facet synovial cyst imaging characteristics, presenting symptoms, change in symptoms after the procedure, and whether they underwent a subsequent operation. We also assessed long-term outcomes from the medical records and via follow-up telephone surveys with patients.

RESULTS: Direct CT-guided lumbar facet synovial cyst puncture was technically successful in $98 \%$ of procedures. At first postprocedural follow-up, $86 \%$ of patients had a complete or partial symptomatic response. During a mean follow-up of 49 months, $56 \%$ of patients had partial or complete long-term relief without the need for an operation; $44 \%$ of patients underwent an operation. Patients with calcified, thick-rimmed, or low T2 signal intensity cysts were less likely to respond to the procedure and more likely to need an operation.

CONCLUSIONS: CT-guided direct lumbar facet synovial cyst aspiration and fenestration procedures are safe, effective, and minimally invasive for symptomatic treatment of lumbar synovial facet cysts. This procedure obviates an operation in a substantial number of patients, even at long-term follow-up, and should be considered before surgical intervention.

ABBREVIATION: LFSC = lumbar facet joint synovial cyst

umbar facet joint synovial cysts (LFSCs) are synovial lined Loutpouchings that arise from the facet joint capsule. ${ }^{1,2}$ While LFSCs may occur at any lumbar level, they most commonly occur at the L4-L5 facets in degenerative facet arthropathy. ${ }^{1,3-5}$ LFSCs have been reported to be present in $0.7 \%-2.0 \%$ of lumbar crosssectional imaging studies. ${ }^{6,7}$ On imaging, LFSCs are well-circumscribed masses contacting or in immediate proximity to the facet joint. Cysts typically demonstrate a thin or thick hypointense signal rim on MR imaging. The internal cyst signal is most commonly T1 hypointense and T2 hyperintense, though these fea-

Received May 1, 2017; accepted after revision August 19.

From the Department of Radiology and Biomedical Imaging, University of California, San Francisco, San Francisco, California.

Paper previously presented at: American Society of Neuroradiology Annual Meeting and the Foundation of the ASNR Symposium, April 25-30, 2015; Chicago, Illinois.

V.N. Shah and N.D. von Fischer contributed equally to this work.

Please address correspondence to Vinil Shah, MD, 505 Parnassus Ave, L352, San Francisco, CA 94143-0628; e-mail: vinil.shah@ucsf.edu

http://dx.doi.org/10.3174/ajnr.A5428 tures can be variable on the basis of intracystic protein content, blood products, calcification, or gas. ${ }^{5,6}$

LFSCs may present with radiculopathy secondary to inflammation or encroachment on nerve roots within the spinal canal or neural foramina or with axial low back pain when arising as a sequela of facet disease. Traditional management consists of laminectomy and surgical resection of the cysts, but in segmental instability or symptomatic spondylolisthesis, facetectomy and posterior spinal fusion are also required. ${ }^{8,9}$ Fluoroscopically guided cyst inflation and indirect rupture have been described as a minimally invasive treatment for symptomatic LFSCs with variable outcomes (23\%-72\% success rates) and follow-up periods (6-44 months). ${ }^{5,7,10-14}$ More recently, direct CT-guided LFSC injection and rupture in a small number of patients have also been described for nonsurgical management. ${ }^{15}$

The aim of our study was to evaluate the effectiveness of direct CT-guided aspiration and fenestration of LFSCs by assessing short-term postprocedural symptom relief and whether an operation could be avoided during long-term follow-up. As a second- 

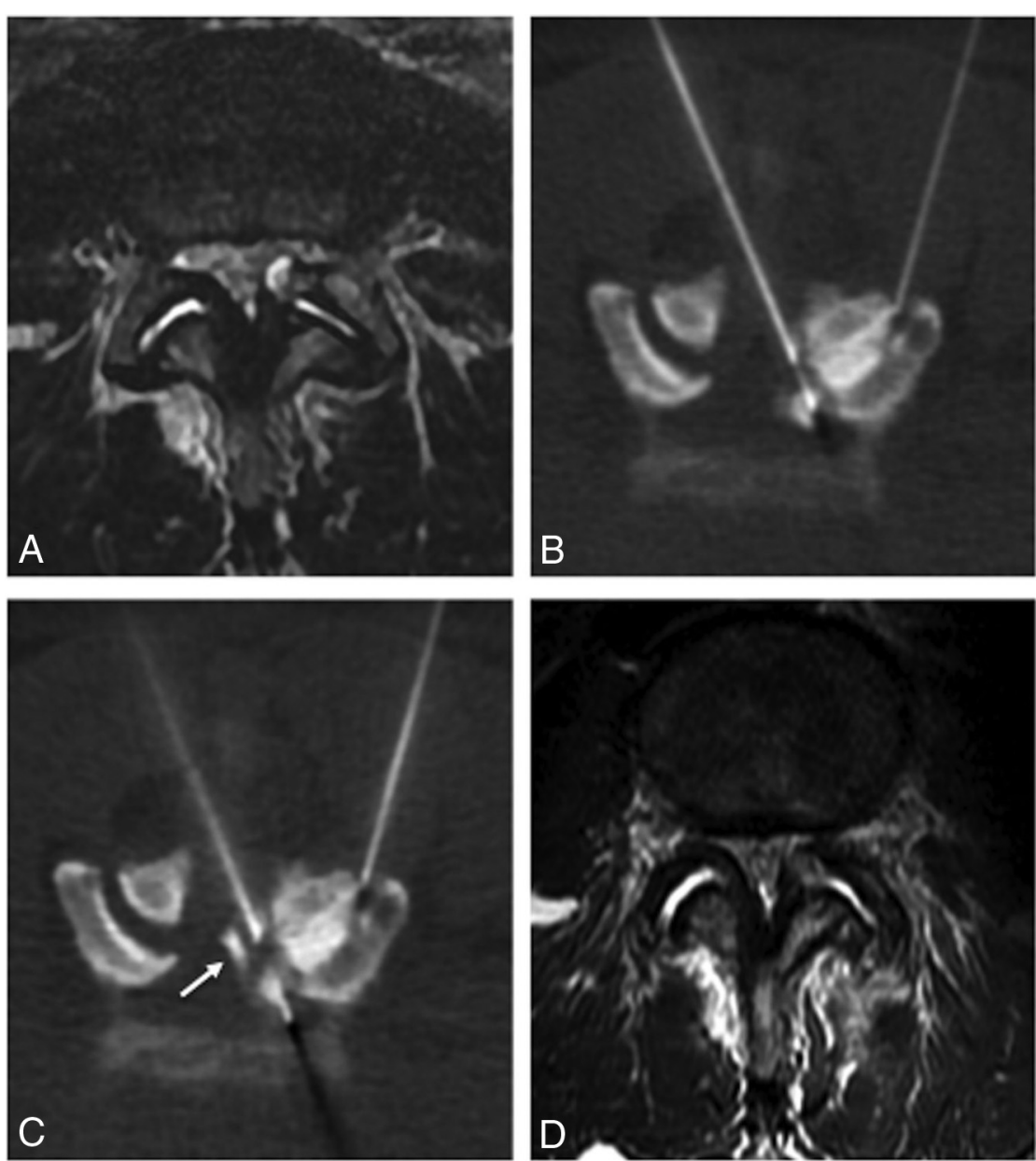

FIG 1. Successful CT-guided cyst puncture, aspiration, and fenestration. A, Axial T2-weighted MR image demonstrates bilateral L4 5 facet synovitis and a thin-rimmed T2-hyperintense cyst arising from the left $L 4-5$ facet joint. $B$, Intraprocedural CT image shows contrast opacification of the cyst via injection into a 22-ga spinal needle placed within the left L4-5 facet joint (step 1). A second 22-ga spinal needle has been advanced coaxially into the cyst via a translaminar approach (step 2). C, CT image obtained after cyst aspiration and repeat fenestration demonstrates successful cyst perforation with leakage of contrast into the epidural space (arrow, step 3). D, Follow-up MR imaging shows resolution of the treated cyst.

ary objective, we sought to determine whether the patient's presenting symptoms, LFSC imaging characteristics, or injection technique could be used to predict response.

\section{MATERIALS AND METHODS \\ Study Design}

The institutional review board approved our retrospective review of patients who underwent CT-guided LFSC aspiration and fenestration during a 10-year period from 2006 to 2016. Patients were identified using a search of our radiology information system. We performed a detailed medical record review and recorded patient demographics, presenting symptoms, change in symptoms at initial follow-up, and whether an operation was performed during the 6-month postprocedural interval. Additionally, we reviewed the most recent preprocedural MR imaging to identify the level of the LFSC, determine its size using the largest diameter on either sagittal or axial images, and record its T2signal characteristics. We also recorded CT characteristics of the cyst from images obtained during the procedure. For those pa- tients in whom a postprocedural MR imaging or CT was available, we also noted whether the cyst persisted or recurred. Finally, we assessed long-term outcomes as documented in the patient's medical record and via a follow-up telephone survey.

\section{Cyst Aspiration and Perforation Technique}

All CT-guided LFSC aspiration and fenestration procedures were performed at our outpatient imaging center by 1 of 6 neuroradiologists experienced in spine intervention (range of experience, 3-34 years; mean, 13 years). The patient was placed in the prone position, and limited low-dose CT of the lumbar spine was performed for procedural planning. The overlying skin was marked, prepped, and draped using an aseptic technique. Local anesthesia was achieved with lidocaine $1 \%$ buffered with $8.4 \%$ sodium bicarbonate. Moderate conscious sedation was provided for most procedures. Intermittent low-dose CT was used for needle guidance. In all except 8 procedures, the parent facet joint was first accessed. One to two milliliters of a mixture of bupivacaine $0.75 \%$ and either Celestone $6 \mathrm{mg} / \mathrm{mL}$ (betamethasone sodium phosphate) or Kenalog $40 \mathrm{mg} / \mathrm{mL}$ (triamcinolone acetonide) combined with a small volume of Omnipaque 240 (iohexol; GE Healthcare, Piscataway, New Jersey) was injected to treat underlying facet arthropathy and to opacify the associated synovial cyst. Then, the LFSC was directly accessed via a contralateral interlaminar or ipsilateral transforaminal approach (depending on whether the cyst was intracanalicular or foraminal) using either a 19-, 20-, 21-, 22-, or 25-ga spinal or Chiba needle (Cook Medical, Bloomington, Indiana). After we confirmed the position of the needle tip within the LFSC, aspiration was first performed followed by repetitive forward-and-back needle motion to fenestrate the cyst wall (Fig 1). CT was then used to confirm successful decrease in the size of the LFSC and/or leakage of previously administered contrast into the epidural space. In all except 10 procedures, a separate transforaminal/interlaminar epidural injection using 1-2 $\mathrm{mL}$ of the steroid and anesthetic mixture was also performed at the same level to concurrently treat the patient's radiculopathy.

After the procedure, the patients were observed for at least 1 hour before discharge. The referring physician, most often an orthopedic surgeon, neurosurgeon, or pain management specialist, assessed postprocedural changes in symptoms and the decision to pursue additional therapy during short-interval follow-up. 
Table 1: Demographic data

\begin{tabular}{lccc}
\hline & Responders & Nonresponders & $\boldsymbol{P}$ Value \\
\hline Age (mean) (yr) & $61.5(11.4)$ & $59.3(8.4)$ & $.65^{\mathrm{a}}$ \\
Sex (No.) (\%) & & & $.34^{\mathrm{b}}$ \\
$\quad$ Male & $21(36)$ & $1(17)$ & \\
$\quad$ Female & $37(64)$ & $5(83)$ & \\
Presenting symptoms & & & $.58^{\mathrm{b}}$ \\
$\quad$ (No.) (\%) & & & \\
$\quad$ Radiculopathy & $27(47)$ & $2(33)$ & \\
$\quad$ Axial back pain & $4(6)$ & $0(0)$ & \\
Both & $27(47)$ & $4(67)$ & \\
\hline
\end{tabular}

a $P$ value based on the 2-sample $t$ test of the difference between means.

${ }^{b} P$ value based on a $\chi^{2}$ test of the difference in proportions.

\section{Statistical Analysis}

For analysis, patients were categorized as responders (those with partial or complete symptom relief) or as nonresponders (those with no change or exacerbation of symptoms based on their first postprocedural visit with referring physicians). We tested for differences between the responders and nonresponders using the $t$ test for LFSC size and patient age and the $\chi^{2}$ test for sex, presenting symptoms, imaging characteristics, and injection technique. Regarding imaging characteristics, we considered cyst level, degree of canal stenosis, T2 signal intensity, and the presence of cyst calcification. $P$ values of $<.05$ were considered statistically significant and $P$ values of $\geq .05$ and $\leq .1$ indicated a trend toward significance. Statistical analysis was performed by using STATA data analysis and statistical software (StataCorp, College Station, Texas).

\section{RESULTS}

\section{Study Population}

During the 10-year study period, 64 patients underwent CTguided LFSC aspiration and fenestration and were included in this investigation. The study population included 22 men and 44 women with an average age of 61 years (range, $36-86$ years). Most patients presented with radiculopathy $(46 \%)$ or radiculopathy with axial low back pain (48\%). Three (5\%) patients had a prior operation for LFSC resection and presented with recurrent symptomatic cysts. There were no significant associations between a successful outcome and age, sex, or symptoms at presentation $(P>.1)$ (Table 1).

\section{Imaging Characteristics}

The mean LFSC size was $10 \mathrm{~mm}$ (range, 5-21 mm). The most commonly treated level of LFSC was L4-L5 (58\%). There was no significant difference in the cyst size, level, or degree of central canal stenosis between the responders and the nonresponders or between those patients who required a subsequent operation and those managed conservatively $(P>.1)$ (Tables 2 and 3$)$.

Preprocedural MRIs were available for review in 60 (94\%) patients. Most (67\%) patients had cysts that were predominantly T2 hyperintense with a thin T2-hypointense rim. The remaining patients had T2-hyperintense cysts with a thick T2-hypointense rim $(30 \%)$ or cysts that were predominantly $\mathrm{T} 2$ hypointense (3\%). On CT, $28 \%$ of cysts were partially or completely calcified. Patients with thin-rimmed T2-hyperintense or noncalcified cysts were more likely to have a favorable response to the procedure than those patients with thick-rimmed, hypointense, or calcified
Table 2: Differences in imaging characteristics of cysts between responders and nonresponders

\begin{tabular}{lccc}
\hline & & & $P$ \\
& Responders & Nonresponders & Value \\
\hline LFSC size (mean) (SD) (mm) & $10.1(3.7)$ & $9.3(2.5)$ & $.62^{\mathrm{a}}$ \\
Cyst level (No.) (\%) & & & $.66^{\mathrm{b}}$ \\
L1-L2 & $1(2)$ & $0(0)$ & \\
L2-L3 & $3(5)$ & $1(17)$ & \\
L3-L4 & $6(10)$ & $1(17)$ & \\
L4-L5 & $35(60)$ & $2(33)$ & \\
L5-S1 & $13(22)$ & $2(33)$ & $.70^{\mathrm{b}}$ \\
MRI central canal stenosis & & & \\
$\quad$ (No.) (\%) & $19(33)$ & $2(33)$ & \\
None & $9(15)$ & $0(0)$ & \\
Mild & $11(19)$ & $1(17)$ & \\
Moderate & $19(33)$ & $3(50)$ & \\
Severe & & & \\
MRI signal intensity (No.) (\%) & $39(72)$ & $1(17)$ & \\
High T2 with a thin rim & $13(24)$ & $5(83)$ & \\
High T2 with a thick rim & $2(4)$ & $0(0)$ & \\
Low T2 & & & \\
CT characteristics (No.) (\%) & $14(24)$ & $4(67)$ & \\
Calcified & $44(76)$ & $2(33)$ & \\
Not calcified &
\end{tabular}

a $P$ value based on the 2-sample $t$ test of the difference between means.

${ }^{\mathrm{b}} P$ value based on a $\chi^{2}$ test of the difference in proportions.

Table 3: Differences in imaging characteristics of cysts between patients who required surgery and those who did not at long-term follow-up

\begin{tabular}{lccc}
\hline & Surgery & No Surgery & $\boldsymbol{P}$ Value \\
\hline LFSC size (mean) (SD) (mm) & $10.5(3.9)$ & $9.5(3.3)$ & $.32^{\mathrm{a}}$ \\
MRI signal intensity (No.) (\%) & & & $.07^{\mathrm{b}}$ \\
High T2 with a thin rim & $11(48)$ & $24(78)$ & \\
High T2 with a thick rim & $11(48)$ & $6(19)$ & \\
Low T2 & $1(4)$ & $1(3)$ & \\
CT characteristics (No.) (\%) & & & $.003^{\mathrm{b}}$ \\
Calcified & $12(48)$ & $4(12)$ & \\
Not calcified & $13(52)$ & $28(88)$ & \\
\hline
\end{tabular}

a $P$ value based on the 2-sample t test of the difference between means.

${ }^{b} P$ value based on a $\chi^{2}$ test of the difference in proportions.

cysts $(P<.05)$ (Table 2$)$. Patients with calcified cysts were more likely to have required an operation at long-term follow-up than patients with noncalcified cysts $(P=.003, \mathrm{OR}=6.5)$ (Table 3$)$. There was a trend toward a greater need for surgical management in patients with thick-rimmed or hypointense cysts as opposed to those with thin-rimmed T2-hyperintense cysts $(P=.07)$.

Postprocedural MR imaging or CT studies were available in 26 (41\%) patients. In most (77\%) of these patients, the treated cyst was noted to be smaller or had resolved; in 5 patients (19\%) the cyst was unchanged, and in 1 patient $(3.9 \%)$ the treated cyst was noted to have increased in size.

\section{Injection Technique and CT Fenestration Success}

Direct CT-guided LFSC puncture and fenestration were technically successful in 81 (98\%) procedures performed with no complications. In 1 patient, the calcified nature of bilateral L5-S1 cysts precluded direct puncture (Fig 2). In another, a foraminal synovial cyst could not be successfully accessed due to overlying bone. Most (94\%) calcified cysts, however, were successfully fenestrated. Facet joint injections were performed before direct cyst fenestration in most of these patients. The ipsilateral facet joint was injected in $56(88 \%)$ patients to opacify the LFSC and to treat under- 


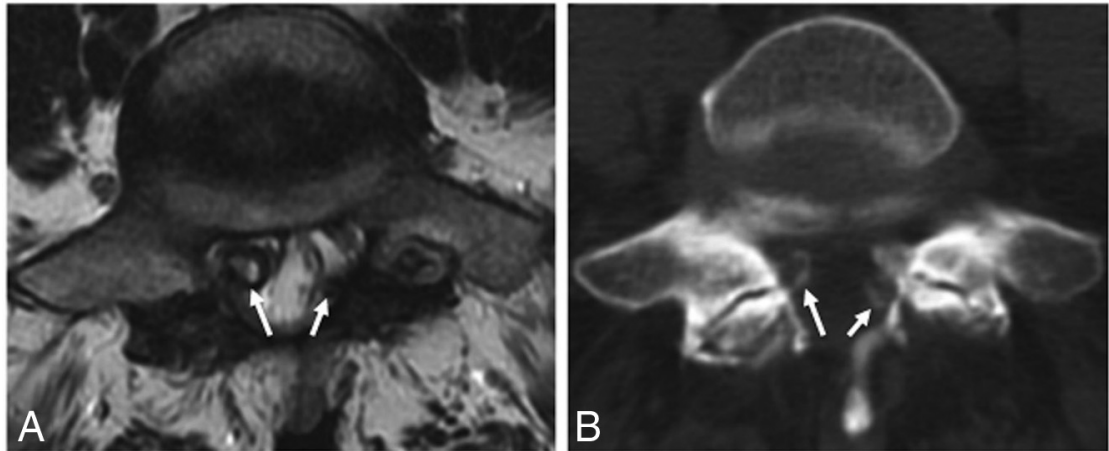

FIG 2. Calcified synovial cysts. A, Axial T2-weighted MR image shows severe bilateral L5-S1 facet arthrosis with thick-rimmed facet cysts (arrows), which result in severe lateral recess narrowing on the right and indentation of the thecal sac on the left. $B$, Axial CT image shows peripheral calcification of both cysts (arrows). Direct cyst puncture and fenestration were not performed in this patient, who instead underwent CT-guided facet injections and nerve blocks and ultimately required an operation.

lying facet arthropathy. The average volume injected in each facet joint was $1.2 \pm 0.9 \mathrm{~mL}$. Concurrent, interlaminar, or transforaminal epidural steroid injections were performed in 54 (84\%) patients to treat associated radiculopathy. There was no significant association between a successful outcome (or subsequent need for an operation) and concurrent facet or epidural steroid injection or needle gauge used during the procedure $(P>.1)$.

\section{Patient Outcomes}

Postprocedure outcome data at first clinical follow-up were available in 61 (95\%) patients. Long-term follow-up data were available for 57 (89\%) treated patients. Mean long-term follow-up was 49 months (range, 2-136 months; median, 44 months). At first postprocedural clinical follow-up, $55(86 \%)$ patients had a partial or complete symptomatic response to CT-guided LFSC aspiration and fenestration. Conversely, $4(6 \%)$ patients had no change in symptoms while $2(3 \%)$ patients reported mild worsening of pain. There were no procedure-related complications. Seventeen (27\%) patients underwent repeat procedures for recurrent symptoms, though they were satisfactorily managed by repeat percutaneous fenestration in two-thirds of cases. Most (76\%) of the repeat patients underwent 1 additional cyst-fenestration procedure; 4 patients required 2 additional cyst-fenestration procedures. Three patients who required repeat procedures had multiple cysts. Two patients underwent repeat cyst fenestration for cysts that recurred after surgical resection. There were no significant differences in imaging features of cysts in patients who required repeat procedures and those who did not $(P>.1$ for cyst size, calcification, T2 signal, level of stenosis, and follow-up MR imaging findings).

Within 6 months following CT-guided LFSC treatment, 18 (30\%) patients underwent an operation for either persistent or recurrent symptoms. At long-term follow-up, 25 (44\%) patients underwent an operation for recurrent symptoms. Patients who needed repeat CT procedures for symptomatic relief were more likely to require an operation at long-term follow-up $(P=.007)$. Three patients needed repeat surgery for recurrent symptoms that could not be managed conservatively. Most of the patients who required surgical management for symptom relief underwent minimally invasive decompression (laminectomy or laminotomy) and cyst resection (61\%), and only 39\% of patients had fusion. The 3 patients who had repeat surgery required fusion. No cyst recurrence was noted in fused patients. The mean time between the CT-guided cyst aspiration procedure and the first operation was $11 \pm 20$ months.

\section{Telephone Follow-Up}

Our institutional review board approved patient telephone contact to obtain follow-up data. Patients were contacted by the first author (V.N.S.), and explicit voluntary consent was obtained before administering a brief telephone survey. Patients who agreed to participate were asked to state their pain scores before and after the procedure, comment on the duration of pain relief, report if they had an operation for the cyst, and were asked if they thought the CT-guided procedure was successful. Forty (63\%) treated patients were contacted by telephone, and all agreed to participate in the survey, at a mean follow-up of 43 months (range, 2-136 months). Seventy-eight percent thought that the CT-guided cyst aspiration procedure was successful. Eighty-seven percent would recommend the procedure to others. The mean numeric rating score of worst pain before the $\mathrm{CT}$ aspiration procedure on a scale of $0-10$ was $8.2 \pm 1.7$. The mean numeric rating score at long-term follow-up was $1.6 \pm 2.2$. There was no significant difference between preprocedural and long-term follow-up numeric rating scores between patients who underwent an operation and those who only underwent the CT-guided fenestration procedure $(P>.1)$.

\section{DISCUSSION}

Our study describes a novel CT-guided, minimally invasive technique for managing symptomatic LFSCs as an alternative for surgical resection with a technical success rate of $98 \%$ and no procedural complications. Most (86\%) patients in our study had at least a partial response at short-term follow-up. More than half (56\%) of our patients did not need an operation at long-term follow-up. Most of the patients who were contacted via phone at long-term follow-up thought the procedure was a success.

In the spine, synovial cysts arise as outpouchings of synovial tissue from the facet joint capsule, which may be symptomatic when located within the spinal canal or neural foramina. Facet joint cysts are more common in the lumbar regions and usually arise at the L4-L5 level. ${ }^{1,3-5}$ Previous authors have postulated that the relatively increased mobility, propensity for degenerative facet arthropathy, and spondylolisthesis at L4-L5 account for the higher frequency of LFSCs., ${ }^{4,13}$ On imaging, LFSCs are well-circumscribed masses contacting or in immediate proximity to the facet joint. Cysts typically demonstrate a thin or thick hypointense signal rim on MR imaging. Internal cyst signal is most commonly $\mathrm{T} 1$ hypointense and $\mathrm{T} 2$ hyperintense, though these features can be variable on the basis of intracystic protein content, blood products, calcification, or gas. ${ }^{5,6}$ 


\section{Percutaneous Management: Fluoroscopy versus CT Guidance}

Minimally invasive, image-guided, management of LFSCs has previously been described. Fluoroscopically guided treatments that have been reported include intra-articular facet injections, cyst aspirations, and predominantly indirect cyst ruptures via parent facet joint access. ${ }^{12-13,16,17}$ Parlier-Cuau et al ${ }^{16}$ reported 30 patients with LFSCs who were treated with fluoroscopically guided facet joint steroid injections. At 6-month follow-up, only $33 \%$ of patients had a favorable response and almost half of the treated patients underwent an operation. In a large study of 101 patients who underwent fluoroscopically guided LFSC rupture (Martha et $\mathrm{al}^{12}$ ), cyst rupture was technically successful in $81 \%$ of cases, and $46 \%$ of patients were free of pain at a mean follow-up of 3.2 years. In that study, cyst rupture was attempted via intraarticular injection of local anesthetic and/or steroid with contrast into the parent facet joint under increasing pressure until the cyst ruptured. Compared with the results described in our study, a larger proportion of their patients had recurrence of symptoms necessitating surgical resection of the LFSC, which may be attributable to differences in procedural techniques. Most interesting in their study, patients whose cysts were successfully ruptured reported higher levels of pain-related disability 3 years postinjection compared with patients whose cysts were not ruptured. We hypothesize this difference may be partly related to neuronal compression following forceful cyst expansion and ultimately rupture.

More recently, investigators have used CT for image-guided spine procedures, including treating LFSCs using both direct and indirect cyst-rupture techniques. ${ }^{5,15,18}$ The indirect technique is similar to that described for fluoroscopically guided cyst inflation and forceful pressurization to achieve cyst rupture. Amoretti et $\mathrm{al}^{18}$ used CT guidance to directly rupture LFSCs in 120 patients, which was technically successful in $77 \%$. Their technique consisted of direct cyst puncture followed by injection of up to $3 \mathrm{~mL}$ of steroid and anesthetic mixture to forcefully rupture the cyst. After $\geq 1$ injection, $75 \%$ of patients were free of pain at 12 months. In another CT-guided study, Ortiz and Tekchandani ${ }^{15}$ performed $\geq 1$ direct percutaneous CT-guided LFSC rupture in 20 patients and reported a $90 \%$ success rate after a mean follow-up of 18 months. Their technique consisted of direct cyst puncture, followed by cyst aspiration, and attempted cyst rupture via injection of 1-3 mL of steroid and anesthetic mixture. These studies demonstrate that the outcome of percutaneous LFSC treatment varies depending on the injection technique, imaging technique used, and follow-up, with good technical success and short-term pain relief and variable longer term outcomes.

The advantages of CT guidance include precise visualization of the cyst, facilitating direct puncture while avoiding critical structures such as thecal sac and exiting nerve. Compared with direct cyst puncture, the indirect cyst rupture technique has limited success in patients in whom it is not possible to access the parent facet joint, in whom the cyst does not communicate with the facet, or in cases in which adequate pressurization of the cyst cannot be achieved. The latter limitation may occur in cases in which the injectate refluxes out of the posterior joint capsule and in patients who may have thick or calcified cyst walls that are resistant to pressure-induced rupture.

Our CT-guided fenestration technique has not been previously described and differs from other CT-guided techniques that have used forceful pressurization of the cyst to induce cyst rupture after direct cyst puncture. Our back-and-forth fenestration technique likely creates multiple sites of cyst wall disruption, which may delay recurrence compared with cyst rupture under pressure (which likely results in a single fenestration). In our experience, the fenestration technique is far less painful than cyst inflation and forceful pressurization to induce rupture, facilitating faster patient recovery and minimizing the amount of procedural sedation. This technique may also be less likely to cause injury to adjacent nerves, which may occur with rapid cyst inflation. As part of our procedure, we injected a small volume of steroid, anesthetic, and contrast to opacify the cyst and treat facet arthropathy. Our average injectate volume of $1.2 \mathrm{~mL}$ is the lowest among all published studies and contrasts with reported volumes of 3.5-15 mL $\mathrm{mL}^{7,13,14}$ used for indirect cyst rupture.

\section{Surgical Management and Associated Complications}

Surgical resection may be considered for patients with persistent intractable pain and neurologic deficits. As part of a systematic review, Bydon et al ${ }^{19}$ reviewed 82 studies including 966 patients treated with synovial cyst excision with or without concomitant spinal fusion. During a follow-up of 25 months, $22 \%$ of patients had recurrent back pain, $6 \%$ required reoperation, and $78 \%$ of those required spinal fusion for instability and mechanical back pain. Recurrence of synovial cysts at the same level occurred in $1.8 \%$ of patients after decompression alone, but none recurred in patients who underwent decompression with concomitant spinal fusion. The surgical complication rate was $4.8 \%$ and included a dural tear, CSF leak, deep venous thrombosis, and 1 death.

Other complications that have been reported after an operation include worsening postoperative instability (especially after laminectomy without fusion), nonunion following fusion, infection, and postoperative hemorrhage. ${ }^{8}$ Similar to the previously reported surgical literature, $12 \%$ of our patients required re-operation and none of our patients who underwent fusion during the primary operation had cyst recurrence or needed re-operation. The surgical data suggest good overall long-term outcomes after surgical cyst excision with a low recurrence rate, but a nontrivial complication rate. The management of symptomatic lumbar synovial cysts is therefore challenging, regardless of the treatment option.

\section{Imaging Predictors of Success}

Twenty-eight percent of the cysts in our study were calcified on CT, comparable with previous descriptions. ${ }^{20}$ While we were able to successfully fenestrate even calcified cysts, patients with calcified cysts were less likely to respond to our procedure and more likely to require an operation at long-term follow-up. On MR imaging, those patients with thin-rimmed T2 hyperintense cysts, as opposed to thick-rimmed or low-T2 signal cysts, were more likely to have a successful outcome (statistically significant) and were less likely to require an operation (trend). Reasons for this could be that cysts with calcification, hemorrhage, or elevated protein content or cysts with thick fibrous capsules are less likely 
to collapse and more likely to recur, even after successful aspiration and fenestration. These results are consistent with those of Cambron et $\mathrm{al}^{5}$ and Huang et al. ${ }^{13}$ Cambron et al examined the ability of the MR imaging appearance of LFSCs to predict the response to CT-guided indirect cyst rupture. In their study of 110 patients, LFSCs with high or intermediate T2 signal were easier to rupture and were less likely to require an operation. Huang et al reported their experience with percutaneous cyst rupture in 71 patients, $36 \%$ of whom had calcified cysts. In their study, calcified cysts were less likely to result in a technically successful procedure, but there was no effect on long-term outcome or the need for an operation.

\section{Long-Term Follow-Up}

Our mean follow-up of 49 months is the longest reported for percutaneous treatment of LFSCs. At long-term follow-up, 56\% of our patients had sufficient symptomatic relief from the procedure to obviate an operation. This result is similar to or better than those of previous long-term studies ${ }^{5,12,13}$ (follow-up range, $34-44$ months; $43 \%-55 \%$ of patients requiring an operation). However, our mean time to the first operation at 11 months is longer than that reported by studies ${ }^{5,12-13}$ with a similar number of patients, which may be due to the greater technical success achieved by our CT-guided aspiration and fenestration technique.

\section{Study Limitations}

The limitations of our study include its retrospective design with variable follow-up intervals and lack of a control group. We had long-term follow-up data available on $89 \%$ of patients. Our success rate from the procedure would have been lower if all patients lost to follow-up (11\%) had required an operation. Our study design (retrospective observational cohort) without a control group may also have introduced several biases that could have influenced our outcomes. These include, but are not limited to, patient recall bias and placebo effect. Additionally, because an epidural steroid injection was performed concurrently in most patients, it is possible that this may have caused a positive response, rather than the cyst fenestration. We recognize these limitations and think that the results of our study should be further validated with a control group. Study strengths include our large number of patients and long-term follow-up.

\section{CONCLUSIONS}

CT-guided direct LFSC aspiration and fenestration are a unique technique associated with high technical success, low complication rates, and resolution of symptoms. In our experience, with careful patient selection, the procedure obviates an operation in a substantial number of patients and should be considered before surgical intervention.

Disclosures: Christopher P. Hess—UNRELATED: Expert Testimony: various, Comments: miscellaneous medicolegal; Grants/Grants Pending: Quest Diagnostics, GE
Healthcare*; Travel/Accommodations/Meeting Expenses Unrelated to Activities Listed: Siemens. *Money paid to the institution.

\section{REFERENCES}

1. Kao CC, Winkler SS, Turner JH. Synovial cyst of spinal facet: case report. J Neurosurg 1974;41:372-76 CrossRef Medline

2. Knox AM, Fon GT. The appearance of lumbar intraspinal synovial cysts. Clin Radiol 1991;44:397-401 CrossRef Medline

3. Jackson DE, Atlas SW, Mani JR, et al. Intraspinal synovial cysts: MR imaging. Radiology 1989;170:527-30 CrossRef Medline

4. Hsu KY, Zucherman JF, Shea WJ, et al. Lumbar intraspinal synovial and ganglion cysts (facet cysts): ten-year experience in evaluation and treatment. Spine (Phila Pa 1976) 1995;20:80-89 CrossRef Medline

5. Cambron SC, McIntyre JJ, Guerin SJ, et al. Lumbar facet joint synovial cysts: does $\mathrm{T} 2$ signal intensity predict outcomes after percutaneous rupture? AJNR Am J Neuroradiol 2013;34:1661-64 CrossRef Medline

6. Apostolaki E, Davies AM, Evans N, et al. MR imaging of lumbar facet joint synovial cysts. Eur Radiol 2000;10:615-23 CrossRef Medline

7. Allen TL, Tatli Y, Lutz GE. Fluoroscopic percutaneous lumbar zygapophyseal joint cyst rupture: a clinical outcome study. Spine J 2009;9:387-95 CrossRef Medline

8. Lyons MK, Atkinson JL, Wharen RE, et al. Surgical evaluation and management of lumbar synovial cysts: the Mayo Clinic experience. J Neurosurg 2000;93(1 suppl):53-57 CrossRef Medline

9. Khan AM, Synnot K, Cammisa FP, et al. Lumbar synovial cysts of the spine: evaluation of surgical outcome. J Spinal Disorder Tech 2005; 18:127-31 CrossRef Medline

10. Lutz GE, Shen TC. Fluoroscopically guided aspiration of a symptomatic lumbar zygapophyseal joint cyst: a case report. Arch Phys Med Rehabil 2002;83:1789-91 CrossRef Medline

11. Melfi RS, Aprill CN. Percutaneous puncture of zygapophyseal joint synovial cyst with fluoroscopic guidance. Pain Med 2005;6:122-28 CrossRef Medline

12. Martha JF, Swaim B, Wang DA, et al. Outcome of percutaneous rupture of lumbar synovial cysts: a case series of 101 patients. Spine J 2009;9:899-904 CrossRef Medline

13. Huang AJ, Bos SA, Torriani M, et al. Long-term outcomes of percutaneous lumbar facet synovial cyst rupture. Skeletal Radiol 2017;46: 75-80 CrossRef Medline

14. Eshraghi Y, Desai V, Cajigal C, et al. Outcome of percutaneous lumbar synovial cyst rupture in patients with lumbar radiculopathy. Pain Physician 2016;19:E1019-25 Medline

15. Ortiz AO, Tekchandani L. Improved outcomes with direct percutaneous CT guided lumbar synovial cyst treatment: advanced approaches and techniques. J Neurointerv Surg 2014;6:790-94 CrossRef Medline

16. Parlier-Cuau C, Wybier M, Nizard R, et al. Symptomatic lumbar facet joint synovial cysts: clinical assessment of facet joint steroid injection after 1 and 6 months and long-term follow-up in 30 patients. Radiology 1999;210:509-13 CrossRef Medline

17. Shah RV, Lutz GE. Lumbar intraspinal synovial cysts: conservative management and review of the world's literature. Spine J 2003;3: 479-88 CrossRef Medline

18. Amoretti N, Huwart L, Foti P, et al. Symptomatic lumbar facet joint cysts treated by CT-guided intracystic and intra-articular steroid injections. Eur Radiol 2012;22:2836-40 CrossRef Medline

19. Bydon A, Xu R, Parker SL, et al. Recurrent back and leg pain and cyst reformation after surgical resection of spinal synovial cysts: systematic review of reported postoperative outcomes. Spine J 2010;10: 820-26 CrossRef Medline

20. Wang A, Haykal H, Lin J, et al. Synovial cysts of the lumbar spine: CT evaluation. Comput Radiol 1987;11:253-57 CrossRef Medline 